\title{
A prática de terapias alternativas/complementares em adultos pós acidente vascular cerebral em hemisfério esquerdo: relato de experiência
}

\author{
The practice of alternative/complementary therapies in adults after stroke in the left \\ hemisphere: an experience report
}

La práctica de terapias alternativas/complementarias en adultos después de un accidente cerebrovascular en el hemisferio izquierdo: un informe de la experiencia

Thainara Carina Almeida Dezincourt ${ }^{1 *}$, Thaís Mara Silva Dezincourt ${ }^{1}$, Jaciara Pereira de Siqueira ${ }^{1}$, Fabiane Corrêa do Nascimento', Karen Tavares dos Santos ${ }^{1}$, Lília Rocha Pinto', Bruna Leal da Silva ${ }^{1}$,

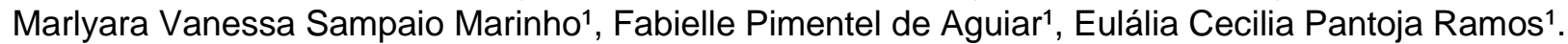

\section{RESUMO}

Objetivo: Descrever os benefícios das terapias alternativas/complementares em uma paciente com limitações pós-AVC. Relato de experiência: Trata-se de um relato de experiência, conduzido com uma paciente cadastrada em uma Unidade Básica de Saúde (UBS) no município de Santarém-PA, nos meses de outubro e dezembro de 2019. Como fonte de dados utilizou-se do prontuário disponibilizado pela enfermeira responsável pelo atendimento da paciente e os diálogos entre as seções de massoterapia para o levantamento de informações comportamentais, histórico familiar e psicológicas. Foram realizadas 13 visitas domiciliares, realizando-se a massoterapia como forma de auxilio no tratamento. Nesta experiência, notouse que ocorreu o ganho de amplitude dos movimentos articulares, melhora na coordenação e aparente relaxamento da musculatura, além de um aumento da motivação, autoestima, bem como da reintegração das atividades de vida diárias e um melhor desempenho das terapias em junção com o tratamento fisioterapêutico e farmacológico. Considerações finais: Por meio das seções de massoterapia foi observado uma melhora progressiva da paciente, o que reforça que a utilização das terapias alternativas em auxílio com o tratamento fisioterapêutico e farmacológico beneficia os pacientes para sua melhor qualidade de vida.

Palavras-chave: Terapias complementares, Massagem, Acidente Vascular Cerebral.

\begin{abstract}
Objective: To describe the benefits of alternative/complementary therapies in a patient with post-stroke limitations. Experience report: This is an experience report, conducted with a patient registered in a Basic Health Unit (UBS) in the municipality of Santarém-PA, in the months of October and December 2019. As a data source, we used the medical record provided by the nurse responsible for patient care and the dialogues between the sections of massage therapy for the survey of behavioral, family and psychological information. Thirteen home visits were carried out, with massage therapy as a form of assistance in the treatment. In this experience, it was noted that there was a gain in range of articular movements, improvement in coordination and apparent relaxation of the muscles, in addition to increased motivation, selfesteem, as well as the reintegration of activities of daily living and a better performance of therapies in junction with physiotherapeutic and pharmacological treatment. Final considerations: Through the massage therapy sections, a progressive improvement of the patient was observed, which reinforces that the use of alternative therapies to assist with physiotherapeutic and pharmacological treatment benefits patients for their better quality of life.
\end{abstract}

Keywords: Complementary therapies, Massage, Stroke.

1 Universidade do Estado do Pará (UEPA), Santarém - PA. *E-mail: naracdezincourt@gmail.com 


\section{RESUMEN}

Objetivo: Describir los beneficios de las terapias alternativas/complementarias en un paciente con limitaciones post ictus. Informe de experiencia: Se trata de un informe de experiencia, realizado con un paciente registrado en una Unidad Básica de Salud (UBS) en el municipio de Santarém-PA, en los meses de octubre y diciembre de 2019. Como fuente de datos, se utilizó la historia clínica proporcionada. por la enfermera responsable de la atención al paciente y los diálogos entre las secciones de masajes para el relevamiento de información conductual, familiar y psicológica. Se realizaron trece visitas domiciliarias, con la terapia de masajes como forma de asistencia en el tratamiento. En esta experiencia, se observó que hubo una ganancia en la amplitud de movimientos articulares, mejora en la coordinación y aparente relajación de los músculos, además de un aumento de la motivación, la autoestima, así como la reintegración de las actividades de la vida diaria. y un mejor desempeño de las terapias en conjunción con el tratamiento fisioterapéutico y farmacológico. Consideraciones finales: A través de las secciones de terapia de masajes, se observó una mejora progresiva del paciente, lo que refuerza que el uso de terapias alternativas para ayudar con el tratamiento fisioterapéutico y farmacológico beneficia a los pacientes por su mejor calidad de vida.

Palabras clave: Terapias complementarias, Masajes, Accidente cerebrovascular.

\section{INTRODUÇÃO}

O Acidente Vascular Cerebral (AVC) é uma patologia, proveniente do episódio de alteração súbita na irrigação sanguínea, caracterizada por sinais clínicos de alteração da função cerebral, de etiologia vascular aguda, permanecendo mais de 24 horas ou levando à morte. Há duas categorias de classificação, o isquêmico por obstrução de vasos sanguíneos e o hemorrágico por ruptura vascular (LIMA ACMACC, et al., 2016).

A categoria de AVC mais prevalente é o isquêmico com percentual de $80 \%$ em comparação ao hemorrágico de $15 \%$. Todavia, o AVC hemorrágico dispõe-se de índices de mortalidade de cerca de 50\% no primeiro mês do evento, de tal forma que se considera a categoria mais crítica das classificações, tendo como característica a sua ocorrência em população mais jovem (SILVA DN, et al., 2019; BARBOSA MAR, et al., 2009; MENOITA EC, 2012).

O Brasil está entre os dez primeiros países com maiores índices anualmente, sendo registrados cerca de 90 mil óbitos por doenças cerebrovasculares. Do mesmo modo, o Sistema Único de Saúde (SUS) registrou cerca de 76.162 mil internações por AVC em 2018 e 2019, em pacientes maiores de 40 anos (BRASIL, 2021).

Em virtude desse episódio cerebrovascular manifesta-se nos pacientes alterações das funções motoras, sensoriais, comportamentais, preceptivas e de linguagem (FREITAS AS, et al., 2017). Neste contexto, se encontra a necessidade de se aplicar técnicas de reabilitação eficazes, à vista disso as Terapias Alternativas/Complementares (TAC), contemplam esse propósito em razão de ser aplicada como meio de prevenção, promoção, manutenção e recuperação da saúde, assim como dispor de um modelo de atenção humanizada e centrada na integralidade do indivíduo, pautados no acolhimento, na escuta e no desenvolvimento de vínculos terapêuticos (BRASIL, 2006).

Dentre as TAC, há a técnica de massagem, que engloba diversas modalidades, dentre elas a massagem terapêutica. Uma terapia tradicional do Oriente, bastante recomendada como tratamento e prevenção em diversas categorias de enfermidades. Pode ser definido como "um conjunto de manipulações e / ou manobras realizadas com as mãos do terapeuta e aplicadas ao corpo humano com objetivos preventivos, higiênicos, terapêuticos, reabilitadores e psicológicos de maneira metódica, ordenada e racional". Com o intuito de promover o relaxamento muscular, nutrição e oxigenação dos tecidos, melhora da circulação sanguínea e do fluxo de drenagem natural dos gânglios linfáticos (RODRíGUEZ-MANSILLA J, 2017; GONDIM SS e ALMEIDA MAPT, 2018). 
Diante disso, este relato de experiência possui como objetivo relatar a experiência das acadêmicas do curso de enfermagem da Universidade do Estado do Pará (UEPA) na realização da Atividade Integradas de Saúde (AIS) que detinha como intuito descrever os benefícios das terapias alternativas/complementares, propriamente a massoterapia, em uma paciente com limitações pós-AVC.

\section{RELATO DE EXPERIÊNCIA}

O curso de Graduação em Enfermagem da Universidade do Estado do Pará busca a formação de profissionais que atendam ao perfil comprometido com a transformação dos modelos assistenciais conforme o que preconiza a Resolução CNE/CES n. 3/2001 - Diretrizes Curriculares Nacionais do Curso de Graduação em Enfermagem (CÂMARA DE EDUCAÇÃO SUPERIOR, 2001). Mediante a isto, o Projeto Pedagógico do Curso de Graduação em Enfermagem, resolução N²666/13 - CONSUN, de 25 de fevereiro de 2014, determina como uma das atividades de caráter obrigatório do curso a realização das Atividades Integradas de Saúde (AIS), no qual, utilizar-se de métodos que favorece o conhecimento crítico-reflexivo dos discentes (UNIVERSIDADE DO ESTADO DO PARÁ, 2014).

Diante disso, a AIS desenvolvido no $4^{\circ}$ semestre do curso de Enfermagem com o eixo temático, Fundamentos Teóricos e Metodológicos de Enfermagem, foi desenvolvido através de grupos formados por discentes com determinados temas específicos dentre os componentes curriculares do semestre, no qual entre os temas abordados há as Terapias Alternativas/Complementares. Nesse sentido, as atividades foram desenvolvidas após uma visita domiciliar em Santarém, Pará, no dia 30 de setembro de 2019, com a presença da enfermeira e agente comunitária da Unidade Básica de Saúde (UBS), assim como orientadora da AIS, em conjunto com as discentes. Dessa forma, constatou-se que a paciente dispusera de alterações da mobilidade corporal que dificulta as suas atividades do cotidiano.

Consoante às observações foram levantados os seguintes dados: Paciente, Z. S. S. sexo feminino, 52 anos, parda, costureira aposentada, nascida no dia 21/04/1967, divorciada, proveniente de Santarém Pará. No dia 19/08/2016 apresentou os primeiros episódios de fraqueza muscular súbita, associado à hemiparesia direita, sendo então, encaminhada para o serviço de urgência e emergência do Hospital Municipal de Santarém, na qual foi diagnosticado AVC Hemorrágico. No período em que esteve hospitalizada realizou tomografia computadorizada de crânio, evidenciando lesão bilateral esquerda.

Atualmente apresenta disartria, alteração no campo de visão e hemiparesia desproporcional de predomínio braquial e podal à direita, deixando sua marcha prejudicada do tipo ceifante com déficit de equilíbrio estático e mão direita em garra com hipertonia. Paciente relata que antes era independente, entretanto após o AVC adquiriu limitações que levaram a não desempenhar serviços laborais e necessitar da assistência dos filhos nas atividades diárias. Faz uso de medicações, possui histórico de etilismo e tabagismo, tendo como início na adolescência e com o abstêmio entre 40 a 45 anos.

Diante da observação foram levantadas hipóteses de solução pelas discentes através de discussão do caso, para assim ser utilizado as TAC apropriadas ao quadro clínico da paciente. Desse modo, com o intuito de minimizar a problemática observada, realizou-se visitas semanais a residência da paciente com a utilização das TAC para o tratamento auxiliar, compreendendo a massoterapia com o uso de óleos corporais.

As visitas para ser aplicadas as seções de massoterapia iniciaram no dia 8 de outubro de 2019, sendo planejado um cronograma de duas visitas por semana com duração de 40 a 60 minutos, com intercalação entre as duplas formadas pelas discentes para a aplicação da terapia. A massagem era realizada com o uso do óleo corporal de amêndoas com macadâmia, aplicando-se em ambos os lados do corpo, inicialmente no lado esquerdo, não afetado pelo AVC, com o objetivo de prover um bem-estar físico e mental (SILVA DN, 2019). Posteriormente aplicava-se a massoterapia no lado direito, área com sequelas evidenciadas do AVC de modo a aumentar a flexibilidade, circulação sanguínea e melhorar os movimentos. Em decorrência da hemiparesia direita, foi constatado a rigidez do membro superior, local em que a massoterapia era mais vigorosa. 
Desta forma, as discentes executaram 13 visitas domiciliares, na qual se observou nas primeiras visitações o comportamento introvertido e inseguro nas afirmações da paciente, de modo que era seu filho que respondia os questionamentos e transmitia as informações para a equipe, porém com a continuidade das visitas a cliente se tornou comunicativa e receptiva. Os resultados da massoterapia foram observados pelas discentes, por meio dos relatos de afirmação de melhora consoante a percepção da cliente e familiares a partir da segunda semana de terapia, na qual relataram ganhos de amplitude de movimentos articulares dificilmente realizados na avaliação clínica inicial.

Concomitante a isto, as discentes notaram uma melhora na coordenação, sobretudo na mão, visto ser perceptível a rigidez do membro superior direito, o qual ficava totalmente contraído, denominado por espasticidade, caracterizando por hipertonia, tônus e clônus muscular (MONTEIRO CBM, et al., 2015). Além disso, houve uma melhora nos aspectos comportamentais como a motivação e autoestima. Após as sessões, verificou-se que os estímulos sensoriais produzidos pela massagem contribuíram para a recuperação da capacidade funcional resultando no relaxamento da musculatura da paciente, permitindo a evolução dos movimentos e bases minimizadoras para a espasticidade.

Logo, a última visita de massoterapia ocorreu no dia 22 de novembro, posteriormente no dia 4 de dezembro de 2019, foi entregue a referência do encaminhamento para a fonoaudióloga do Sistema Único de Saúde (SUS) e uma cesta com itens de beleza, confeccionadas pelas discentes com a finalidade de proporcionar o bem-estar e agradecer pela receptividade e disponibilidade da paciente para a cooperação na realização no projeto. Conjuntamente, houve o reforço para a continuidade das práticas integrativas para ocasionar maiores avanços a longo prazo. Similarmente a paciente verbalizou a sua gratidão pelo trabalho realizado e o avanço alcançado, bem como expressou interesse em prosseguir com as TCA.

\section{DISCUSSÃO}

Os fatores que influenciam na aquisição do AVC, compreende-se em dois grupos: alteráveis e não alteráveis (PEREIRA TMA, et al., 2019). Os fatores de risco modificáveis são aqueles que implicam nas condições que podem ser evitadas conforme o estilo de vida do indivíduo (SOUSA MR, et al., 2017), e os fatores de risco não modificáveis, aqueles que não podem ser tratados em decorrência de ser imutáveis como a idade, gênero, etnia e hereditariedade (RODRIGUES MS, 2017).

Consoante a isso, os fatores de risco evidenciados na cliente compreendem os fatores não modificáveis abrangendo a idade, tratando-se de um dos indicadores mais importante na sua classificação (MARIANELLI M, et al., 2020), pois é um processo natural do ser humano e na medida que ocorre o envelhecimento há uma maior probabilidade de surgir outros fatores de risco, majoritariamente a partir da faixa etária dos 35 anos (MARTINS ERC, et al., 2016; MOURÃO AM, et al., 2017)

Outro ponto a ser considerado são os fatores que poderiam ser modificados pela cliente, retratados antes do AVC, compreendendo o estresse que se configura como outro fator em decorrência do aumento da produção de glóbulos brancos no organismo o que acarreta acréscimo ao risco de AVC em razão de contribuir para obstrução das artérias (OLIVEIRA RR, 2012; RODRIGUES ERS, et al., 2013; HEIDT T, et al., 2014).

Além disso, segundo Cassola B, et al. (2012), os indivíduos que possuem o hábito de ingerir bebidas alcoólicas de três ou mais doses por dia dispõe de uma porcentagem significativa para ocorrência de AVC com idade média de 60 anos. Do mesmo modo, o tabagismo aumenta esse risco, visto que, pode elevar a possibilidade de um dano cerebral (MCCORKINDALE NA, et al., 2016) e favorece o surgimento de aterosclerose (WANG Z, et al., 2017).

Dentre os distúrbios metabólicos a cliente apresenta-se a hipertensão arterial (HAS) que se confere como fator de risco para o Acidente Vascular Cerebral, por propiciar quatro vezes mais chances aos indivíduos hipertensos a desenvolver AVC em comparação aos normotensos da mesma idade (O'DONELL MJ, et al., 2010). A HAS pode levar ao acometimento das artérias cerebrais e levar ao comprometimento de todas as estruturas que compõem as artérias (ARAUJO LPG, et al., 2017). 
No que tange as complicações do AVC e consecutivo dano causado ao cérebro decorrente do episódio, caracteriza-se pela possível alteração da função motora, sensorial, comportamental, preceptiva e de linguagem (FREITAS, AS et al., 2017). Desse modo, foi constatado na cliente hemiparesia contralateral a lesão, disartria, distúrbio do campo visual e cognitivo.

A hemiparesia consiste na paralisia parcial de um hemicorpo (ARAUJO LPG, et al., 2017), caracterizada pela redução do controle motor, fraqueza muscular e alterações no tônus muscular que provocam dificuldades na execução dos movimentos dos membros superiores e inferiores, comumente associado a variações sensoriais, motoras, distúrbios de linguagem e defeito do campo visual (OLIVEIRA HR, 2018).

Dentre as variações sensoriais mais comuns na hemiparesia envolve a insuficiência sensorial superficial que incluem as alterações tátil, térmica e dolorosa (ARAUJO LPG, et al., 2017). Em conformidade com as observações da paciente verifica-se a insuficiência sensorial superficial, com maior obstáculo na sensibilidade tátil do membro superior direito, bem como deficit na mobilidade.

A disartria é o distúrbio das articulações de sons devido a deficiências no controle neuromuscular, resultante de alterações na força, velocidade, amplitude, firmeza, tom de voz ou precisão e duração dos movimentos necessários para a fala (PENNINGTON L, et al., 2016), na qual se determina como uma sequela reagente do AVC e que tendem graus variados de severidade (HUSTAD KC, et al., 2010).

Mediante as sequelas pós-AVC observada na paciente, foi optada pela técnica de massagem terapêutica. No qual a sua aplicação apresenta intuitos preventivos, higiênicos, terapêuticos, reabilitadores e psicológicos (GALLEGO JV, 2009). Constituindo-se de uma técnica manipulativa para o tratamento de determinadas alterações musculares e outros tecidos moles do corpo (CUCCURULLO SJ, et al., 2019).

Verifica-se que a massagem dispõe de inúmeros benefícios dentre eles o aumento da circulação sanguínea nas áreas massageadas e o decorrente alívio da dor (ALI A, et al., 2017). Ademais, proporciona indiretamente uma atenuação dos níveis de estresse, auxiliando em casos de ansiedade e depressão (ALI A, et al., 2017) por produzir relaxamento muscular e bem-estar geral (ALI A, et al., 2017). Compreendendose em uma ação mecânica com diversos ritmos aplicados inicialmente sobre a pele, atingindo os músculos, os vasos sanguíneos e linfáticos aumentando o metabolismo (GONDIM SS, ALMEIDA MAPT, 2018).

Além disso, promove a vitalidade e flexibilidade dos tecidos cutâneos devido à dilatação dos vasos capilares e ampliação do fluxo sanguíneo na intraderme. E conforme a aplicação acarreta um estímulo superficial, consoante a velocidade e a fricção na sua prática, proporcionando um relaxamento da musculatura na área afetada devido à eliminação da tensão muscular (COSTA FS, et al., 2017).

Diante disso, as TAC viabilizam uma melhora na reabilitação física, social e mental do indivíduo, pois possibilita uma vida mais confortável, ativa e produtiva dentro das limitações da doença (PINTO RFS, et al., 2014). De modo que, o processo de reabilitação do indivíduo que sofreu o AVC deve ser o mais independente possível em um espaço de tempo reduzido, que será determinado conforme as limitações provocadas pelo AVC e a disposição do paciente para adoção das estratégias e recursos aplicados (CUNHA MGT, 2014).

Dessa forma, o paciente deve ser acompanhado por uma equipe multidisciplinar, que compreende os profissionais da Medicina, Enfermagem, Fisioterapia, Terapia Ocupacional, Fonoaudiologia e Serviço Social (LANGSTAFF C, et al., 2014). Sendo assim, necessário a equipe dispor de um tratamento convergente, bem como é pertinente para o auxílio da reabilitação a aplicação de Terapias Alternativas/Complementares (TAC), que é regulamentada no sistema Único de Saúde através da Portaria Ministerial n.․ 971 em maio de 2006, assim como do n. 1.600, de 17 de julho de 2006, constituindo a Política Nacional de Práticas Integrativas e Complementares (BRASIL, 2006).

De forma que sua aplicação mediante os profissionais da enfermagem é reconhecida pela resolução 0500, de março de 2015 do COFEN, desde que realize o curso reconhecido por instituição de ensino ou entidade congênere, com carga horária mínima de 360 horas, assim o enfermeiro torna-se especializado e 
considerado um terapeuta alternativo/complementar (BRASIL, 2015). Essa especialização beneficia o tratamento e o paciente dado que constituem em procedimentos não invasivos e mais prazerosos (CUNHA MGT e NOVO A, 2014).

O resultado do estudo fortalece as evidências na literatura existente que mostra o benefício das terapias complementares na reabilitação de pacientes pós-AVC, com ênfase na massoterapia e a escuta no cuidado de enfermagem. A massoterapia aliada ao tratamento medicamentoso promove uma melhor qualidade de vida, pois disponibiliza um relaxamento muscular, com ganhos de amplitude de movimentos articulares, melhora na coordenação dos membros afetados pela hemiparesia e nos aspectos comportamentais. Além disso, devido ao grande número de limitações físicas e cognitivas após o evento, observou-se a necessidade de uma equipe multidisciplinar no acompanhamento da paciente e uma rede de apoio familiar para a continuidade do cuidado prolongado, bem como neste caso verificou-se a importância do início da reabilitação o mais precoce possível após o evento.

\section{REFERÊNCIAS}

1. ALI A, et al. Massage therapy and quality of life in osteoarthritis of the knee: A qualitative study. Pain Medicine, 2017; 18: $1168-1175$.

2. ARAUJO LPG, et al. Principais fatores de risco para o acidente vascular encefálico e suas consequências: uma revisão de literatura. Revista Interdisciplinar do Pensamento Científico, 2017; 3.

3. BARBOSA MAR, et al. Prevalência da hipertensão arterial sistêmica nos pacientes portadores de acidente vascular encefálico, atendidos na emergência de um hospital público terciário. Revista da Sociedade Brasileira de Clínica Medica, 2009; 7: 357-360

4. BRASIL. Ministério da Saúde. Portaria n. 971, de 03 de maio de 2006. Diário Oficial da União. Brasília (DF)

5. BRASIL. Conselho Federal de Enfermagem. Resolução COFEN 0500. Estabelece e reconhece as Terapias Alternativas como especialidade e/ou qualificação do profissional de Enfermagem. In: Conselho Regional de Enfermagem. Documentos básicos de enfermagem. São Paulo (SP); 2015.

6. BRASIL. Ministério da Saúde. Banco de dados do Sistema Único de Saúde- DATASUS. Disponível em: http://tabnet.saude.sp.gov.br/deftohtm.exe?tabnet/ind43_matriz. Acesso: 13 de fevereiro de 2021.

7. CÂMARA DE EDUCAÇÃO SUPERIOR, Conselho Nacional Educação. Resolução nํ 3, de 7 de novembro de 2001. Brasília, 2001.

8. CASSOLA B, et al. Heavy alcohol intake and intracerebral hemorrhage: characteristics and effect on outcome. Neurology, 2012; 79: 1109-1115.

9. COSTA FS, et al. Acupuntura No Tratamento Da Fibromialgia: Revisão da Literatura. Revista Visão universitária, 2017; 1.

10. CUNHA MGT, NOVO A. Cuidados de Enfermagem de Reabilitação no doente com AVC isquémico e a demora média de internamento hospitalar. Dissertação (Mestrado em Enfermagem de Reabilitação) - Instituto Politécnico de Bragança. Escola Superior de Saúde, Bragança, 2014; 117 p.

11. CUCCURULLO SJ. Physical medicine and rehabilitation board review. Springer Publishing Company, $2019 ; 1024$ p.

12. FREITAS AS, et al. JOGO EDUCATIVO SOBRE ACIDENTE VASCULAR CEREBRAL PARA PRÉADOLESCENTES. Mostra Interdisciplinar do curso de Enfermagem, 2017; 2.

13. GALLEGO JV. Manual professional del massaje. Paidotribo, 2009; 1: $650 \mathrm{p}$.

14. GONDIM SS, ALMEIDA MAPT. Os efeitos da massagem terapêutica manual em pacientes com a síndrome da fibromialgia. ID on line REVISTA DE PSICOLOGIA, 2018;12: 336-354.

15. HEIDT T, et al. Chronic variable stress activates hematopoietic stem cells. Nature Medicine, 2014; 20: 754-758.

16. HUSTAD KC, et al. Classification of speech and language profiles in 4-year-old children with cerebral palsy: $A$ prospective preliminary study. 2010.

17. LANGSTAFF C, et al. Enhancing Community-Based Rehabilitation for Stroke Survivors: Creating a Discharge Link. Topics in stroke rehabilitation, 2014; $21: 510-519$.

18. LIMA ACMACC, et al. Diagnósticos de enfermagem em pacientes com acidente vascular cerebral: revisão integrativa. Revista Brasileira de Enfermagem, 2016; 69: 785-792.

19. MARIANELLI M, et al. Principais fatores de risco do avc isquêmico: Uma abordagem descritiva. Brazilian Journal of Health Review, 2020; 3: 19679-19690.

20. MARTINS ERC, et al. Estudo epidemiológico sobre acidente vascular encefálico em uma clínica escola de Fisioterapia. Revista de saúde pública do Paraná, 2016;17:32-38.

21. MCCORKINDALE NA, et al. The effects of chronic smoking on the pathology of alcohol-related brain damage. Alcohol, 2016; 53: 35-44.

22. MENOITA EC. Reabilitar a pessoa idosa com AVC: Contributos para um envelhecer resiliente. Lusociência, 2012.

23. MONTEIRO CBM, et al. Paralisia Cerebral: teoria e prática. Plêaide. 2015;1: 385- 97

24. MOURÃO AM, et al. Perfil dos pacientes com diagnóstico de AVC atendidos em um hospital de Minas Gerais credenciado na linha de cuidados. Revista Brasileira de Neurologia, 2017; 53: 12-16. 
25. O'DONNELL MJ, et al. Risk factors for ischaemic and intracerebral haemorrhagic stroke in 22 countries (the INTERSTROKE study): A case-control study. The Lancet, 2010; 376: 112-123.

26. OLIVEIRA HR. BENEFICIOS DA FISIOTERAPIA NO ESTÁGIO CRÔNICO DE DISFUNÇÕES MOTORAS E FUNCIONAIS PÓS ACIDENTE VASCULAR CEREBRAL. Dissertação (Conclusão do Curso), 2018.

27. OLIVEIRA RR. Análise dos fatores de risco associados ao acidente vascular encefálico em adultos jovens. Goiânia. Monografia (Fisioterapia) - Pontifícia Universidade Católica de Goiás, Goiás, 2012.

28. PENNINGTON L, et al. Speech therapy for children with dysarthria acquired before three years of age. Cochrane Database of Systematic Reviews, 2016.

29. PEREIRA TMA, et al. Avaliação do perfil dos fatores de risco para Acidente Vascular Cerebral: estudo observacional. Revista Pesquisa em Fisioterapia, 2019; 9:37-44.

30. PINTO RFS, et al. A massoterapia como estratégia de tratamento na redução do quadro álgico e dos níveis de estresse observados em indivíduos residentes na Zona Oeste do Rio de Janeiro. Ciência Atual, $2014 ; 4$.

31. RODRIGUES ESR, et al. Fatores de risco cardiovascular em pacientes com Acidente Vascular Cerebral. Revista Amazônia: Science \& Health, 2013; 1: 21-28.

32. RODRIGUES MS, et al. Fatores de risco modificáveis e não modificáveis do AVC isquêmico: uma abordagem descritiva. Revista de medicina, 2017; 96: 187-192.

33. RODRÍGUEZ-MANSILLA J, et al. Efeitos da aplicação de massagem terapêutica em crianças com câncer: uma revisão sistemática. Rev. Latino-Americana de Enfermagem, 2017; 25.

34. SILVA DN, et al. Cuidados de enfermagem à vítima de acidente vascular cerebral (AVC): Revisão integrativa. Revista Eletrônica Acervo Saúde, 2019; 36: e2156-e2156

35. SOUSA MR, et al. Fatores de risco modificáveis e não modificáveis do AVC isquêmico: uma abordagem descritiva. Revista de Medicina, 2017; 96: 187-192.

36. UNIVERSIDADE DO ESTADO DO PARÁ. Projeto Pedagógico do Curso de Graduação em Enfermagem, Resolução № 2666/13 - CONSUN, de 25 de fevereiro de 2014.

37. WANG Z, et al. Cigarette smoking and adipose tissue: the emerging role in progression of atherosclerosis. Mediators of inflammation, 2017; 2017. 